\title{
The Unique Endosomal/Lysosomal System of Giardia lamblia
}

\author{
Maria C. Touz
}

Additional information is available at the end of the chapter

http://dx.doi.org/10.5772/45786

\section{Introduction}

Endocytosis, which is important for the internalization of nutrients from the plasma membrane as well as extracellular fluids, has been extensively described in mammalian cells and yeast. The protozoan parasite Giardia lamblia (syn. Giardia intestinalis, Giardia duodenalis) is an early branching eukaryote that possesses a reduced membrane network with highly polarized vesicles, denominated peripheral vacuoles (PVs), neighboring the plasma membrane (reviewed by Adam, 2001; Faso and Hehl, 2011; Touz, 2011). This is an important zone of interaction between the parasite and its environment (the host intestine in vivo or the culture medium in vitro), and was shown to be the place involved in fluid-phase and receptor-mediated endocytosis (Gaechter et al., 2008; Rivero et al., 2010). Another characteristic of these PVs is that they contain hydrolytic activity resembling the function performed by lysosomes. These vacuoles have a high lytic capacity and a low luminal $\mathrm{pH}$, both properties of mature lysosomes (Ward et al., 1997; Touz et al., 2002b).

More than twenty years after the first morphological description of the PVs, there is a consensus that these vesicles are rather an unusual combination of endosomal and lysosomal compartments. Conserved markers and mechanisms that govern trafficking to the PVs have been found, but there are particularities that show Giardia as a simplified organism compared with higher eukaryotes. This makes Giardia a unique biological cell model for investigating the minimal machinery employed by a eukaryote to regulate endocytosis and degradation. Here, we will discuss emerging data that are beginning to shed light on the endosomal-lysosomal system in Giardia and the molecules involved in this selective trafficking.

\section{The parasite}

Giardia lamblia is a flagellated protozoan that inhabits the upper small intestine of its vertebrate hosts and is the most common cause of defined waterborne diarrhea worldwide. 
Clinical manifestations of giardiasis vary from asymptomatic infection to acute or chronic disease associated with diarrhea and malabsorption (Adam, 2001). It is the most common cause of diarrheal disease in the United States (Barwick et al., 2000). In developing countries, there is a very high prevalence and incidence of infections, and data suggest that long-term growth retardation can result from chronic giardiasis (Fraser et al., 2000).

Giardia was initially described by van Leeuwenhoek in 1681 from examining his own diarrheal stools under the microscope (Dobell, 1950), but it was not until 1981 that the World Health Organization classified Giardia as a human pathogen. Infections initiate with the ingestion of the cyst forms, which excyst in the upper small intestine of the host. The trophozoites replicate and colonize the intestinal surface and some of them encyst in the lower small intestine after sensing the stimulus for encystation (Lujan et al., 1997; Adam, 2001). Interestingly, during differentiation (encystation/excystation), trophozoites undergo important biochemical and morphological modifications involving the secretory machinery of the cell. Recent studies about these changes have provided new insights into the mechanisms of secretion in this organism, but the molecular events leading to intracellular protein trafficking and secretion in Giardia remain poorly understood or controversial (Lujan and Touz, 2003; Faso and Hehl, 2011).

The trophozoite is between 10 and 15 microns long and 5 microns wide, pear-shaped and cut along the longitudinal axis (pyriform morphology) (Figure 1A). It presents bilateral symmetry, has two diploid nuclei with nuclear membranes, four pairs of flagella and two media bodies consisting of microtubules (Adam, 2001), suggested to be the storage reservoir of microtubules of the cell (Piva and Benchimol, 2004). The trophozoite also possesses a complex cytoskeleton and endomembrane system including the endoplasmic reticulum (ER), which extends symmetrically throughout the cell body, and the PVs located underneath the plasma membrane. In the front half, on the ventral surface, is the adhesive disc which is used to bind to the intestinal epithelium of the host (Elmendorf et al., 2003). The oval cyst size is between 9 and 12 microns and contains four nuclei, the axostyle (structure at the base of the flagella) and remnants of flagella (Figure 1B). It is characterized by a rigid outer wall glycoprotein, composed of proteins and carbohydrate (Jarroll et al., 1989; Manning et al., 1992; Gerwig et al., 2002). The construction of the extracellular cyst wall (CW; Cyst Wall) is of paramount importance because it allows the parasite to persist in fresh water, survive even the action of disinfectants and resist stomach acid in its new host, and then start infection in the gut.
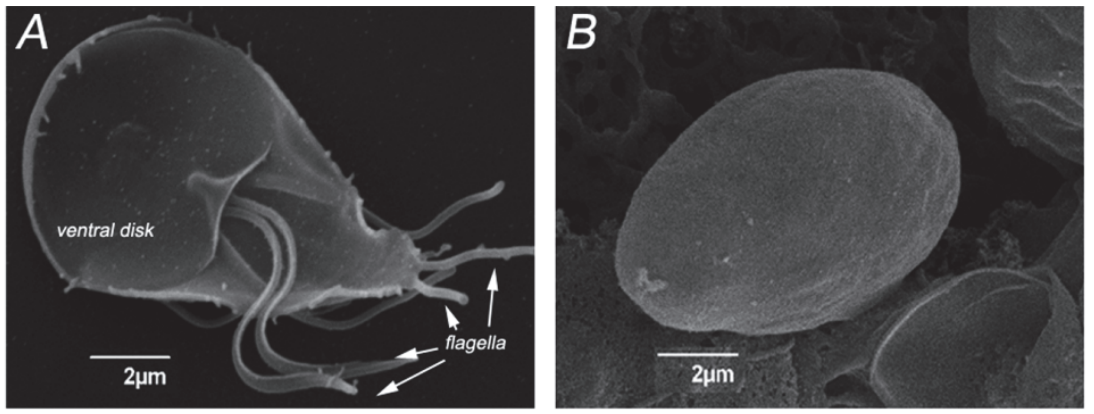

Figure 1. Giardia lamblia stages. (A) Giardia trophozoite, the ventral disk structure and the flagella are highlighted. (B) Giardia cyst. 


\section{Giardia secretory pathway}

Eukaryotic cells have to deal with the fact that, after translation at the ribosomes, most proteins must be specifically targeted from the cytoplasm to the organelle in which they must function. As cellular components became more complex and abundant during evolution, subcellular compartmentalization developed into an essential feature to prevent the inappropriate meeting of certain intracellular components, as well as facilitating efficient ordered reactions (Munro, 2004). To maintain these compartments, cells have evolved mechanisms to ensure that specific proteins are delivered to specific organelles. In most eukaryotes, the Golgi complex serves as a major sorting point in the secretory pathway, selectively targeting proteins and lipids to different organelles ( $\mathrm{Gu}$ et al., 2001). Giardia possesses a distinctive endomembrane system involving the nuclear membrane, the ER, and lysosome-like PVs (Figure 2).
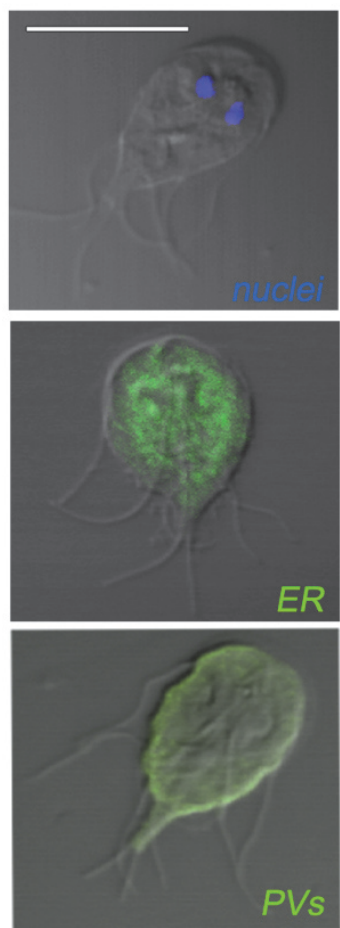

Figure 2. Giardia organelles. Confocal microscopy showing the nuclei, endoplasmic reticulum (ER) and PVs labeled with DAPI (4',6-diamidino-2-phenylindole), anti-BiP mAb, and anti-AP2 mAb, respectively. Bar, $10 \mu \mathrm{m}$.

On the other hand, it lacks other organelles characteristic of higher eukaryotes such as canonical endosomes, lysosomes, mitochondria, peroxisomes, and Golgi apparatus (reviewed by Lujan and Touz, 2003; Hehl and Marti, 2004). In most eukaryotic cells, the Golgi apparatus consists of a series of flattened cisternal membranes forming a stack 
(Ladinsky et al., 2002). The architecture of this organelle is remarkably conserved throughout eukaryotic evolution (Mellman and Simons, 1992); however, a typical Golgi complex with organized and parallel cisternae is not apparent in vegetative Giardia trophozoites. Several pieces of evidence suggest that Giardia trophozoites may possess organelle(s) in which typical Golgi functions take place, even though they do not have a Golgi-like appearance. The fact that both constitutive and regulated mechanisms for protein transport exist in Giardia is an example of Golgi functions, since the sorting and selection process generally occurs in the trans-Golgi network in more complex cells (Gu et al., 2001). The constitutive pathway in Giardia is represented by continuous expression and trafficking to the plasma membrane of the transmembrane-anchored variant-specific surface proteins (VSPs) (Nash, 2002; Marti et al., 2003a; Touz et al., 2005). The regulated pathway takes place only during encystation and associates with the appearance of encystation-specific vesicles (ESVs), which transport cyst wall components to the plasma membrane of the encysting cell and release their content to the cell exterior during cyst wall formation (Reiner et al., 1989; McCaffery and Gillin, 1994; Lujan et al., 1995; Sun et al., 2003). A selective pathway sorting proteins to the endosomal/lysosome membrane system has been recently demonstrated by our group, although Giardia do not possess distinctive endosomes or lysosomes (Touz et al., 2003; Touz et al., 2004; Rivero et al., 2010; Rivero et al., 2011).

Analyses of genes and proteins used for phylogenetic classification indicate that Giardia is in fact one of the earliest branching eukaryotes (Sogin et al., 1989; Hashimoto et al., 1998), but some of the particular cellular characteristics of this organism are probably a result of the secondary loss of complex cell structures, as a consequence of its parasitic life style, rather than the primitive simplicity supposed for early diverging protists (Dacks and Doolittle, 2002; Lujan and Touz, 2003).

\section{The endosomal/lysosomal system of Giardia}

Most eukaryotes have a system of endosomes and lysosomes that mediate the internalization, recycling, transport and breakdown of cellular and extracellular components and facilitate dissociation of receptors from their ligands. Early endosomes (EE) internalize endocytosed proteins to allow for their subsequent return to the cell membrane. Later, conversion of the EEs to late endosomes (LE) takes place, undergoing homotypic fusion reactions, growing in size, and acquiring more intraluminal vesicles. What follows is the fusion of an endosome with a lysosome and maturation of the subsequent endolysosome into lysosome, which constitutes a storage organelle for lysosomal hydrolases at acidic $\mathrm{pH}$, and membrane components. Although this is a highly dynamic system, discrete compartments can be distinguished (Huotari and Helenius, 2011).

In contrast to most eukaryotes, Giardia has highly polarized vacuoles, located underneath the plasma membrane of the dorsal side, which combine some of the characteristics of endosomes and some of lysosomes (Lindmark, 1988; Lanfredi-Rangel et al., 1998; Touz et al., 2004). These PVs, distinguished by their localization, are about $150 \mathrm{~nm}$ in diameter with variable oval shapes and contain a core of low electron density (Figure 3). They are acidic, as 
shown by the uptake of acridine orange and the lysosomal markers Lyso-sensor and Lysotracker (Lanfredi-Rangel et al., 1998; Touz et al., 2002b; Touz et al., 2003). The first description of hydrolase activity in the PVs came from studies in which acid phosphatase activity was tested, showing a cytochemical localization in these vacuoles as well as in the ER and nuclear envelope cisternae (Feely and Dyer, 1987). The presence of hydrolase activities in the PVs was also proved for cysteine proteases and RNases, demonstrating their lysosomal characteristics (Lindmark, 1988; Ward et al., 1997; Touz et al., 2002b). In addition, their potential role in endocytosis was demonstrated by the uptake of exogenous ferritin and Lucifer yellow (Bockman and Winborn, 1968; Lanfredi-Rangel et al., 1998). Pulse-chase experiments with horseradish peroxidase and fluorescent dextran showed an early and persistent labeling of the PVs, suggesting that there is no distinction between early and late endocytic vesicles in Giardia, in contrast to what occurs in higher eukaryotes (LanfrediRangel et al., 1998; Gaechter et al., 2008).

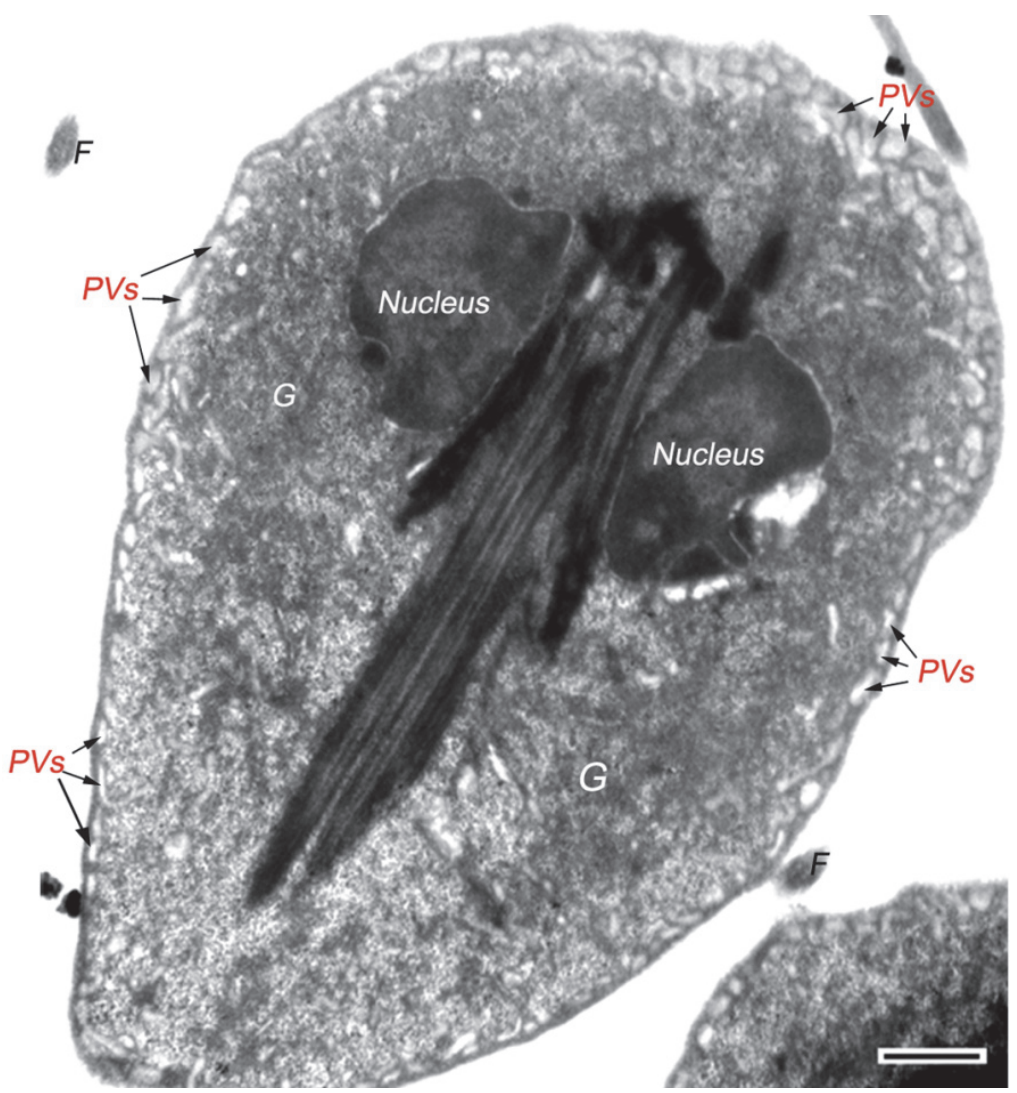

Figure 3. Giardia trophozoite ultrastructure. Electromicrograph of a growing Giardia trophozoite, showing the peripheral vacuoles (PVs) located underneath the plasma membrane (arrows). Nuclei are also denoted. G: electron-dense glycogen deposits. Bar, $0.5 \mu \mathrm{m}$. From Rivero et al., 2010. 
Analysis of fluid-phase endocytosis demonstrated that there is no lateral exchange of fluid phase markers between individual PVs (Gaechter et al., 2008). It was observed that, after internalization, some fluid-phase markers translocated rapidly to the ER or to an associated membrane compartment termed the tubulo-vesicular network (TVN) (Abodeely et al., 2009). Moreover, the presence of protease functions within the TVN, plus 3 -D reconstruction and electron microscopy tomography of trophozoites stained for acid phosphatase and glucose-6-phosphatase, suggest that there might be a connection between some vesicles and profiles of the ER (Lanfredi-Rangel et al., 1998; Abodeely et al., 2009a). However, a recent work showed, by immunofluorescence and 3-D reconstruction, that the ER membranes are found throughout the cytoplasm, but do not permeate the space occupied by PVs (Faso and Hehl, 2011). Recently, it was shown that a mechanism of receptor-mediated endocytosis occurs in this organism, with specific molecules selectively directed to the PVs through a classical endocytic mechanism (Rivero et al., 2010; Rivero et al., 2011). These data suggest that uptake of soluble material into PVs is not selective at this step but is still capable of redirecting specific molecules to the TVN (Hernandez et al., 2007; Abodeely et al., 2009b). In terms of receptor-mediated endocytosis, movement between vesicles could be observed (Figure 4), which suggests, not only that Giardia possesses a refined and conserved mechanism of endocytosis, but also that the PVs population might not be as homogeneous as was thought but rather organized depending on their functions (Rivero \& Touz, unpublished).
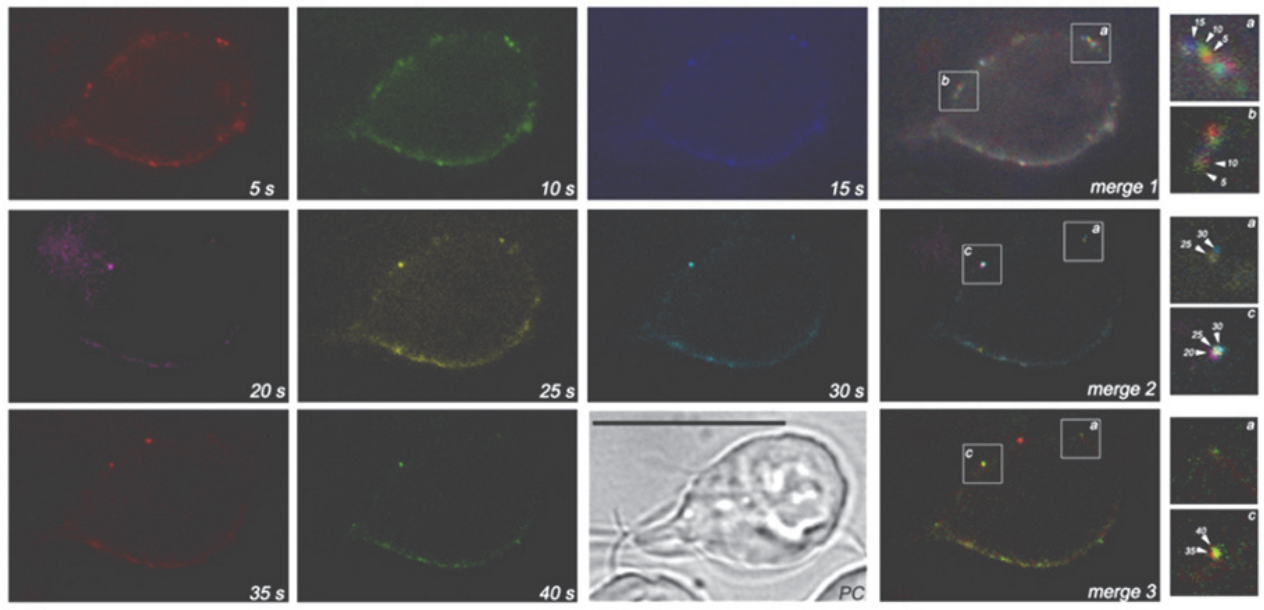

Figure 4. Epifluorescence microscopy shows the internalization and delivery of LDL to the PVs. Eight frames from $5 \mathrm{~s}$ to $40 \mathrm{~s}$ were artificially colored and combined (merge 1-3) to determine the movement of endocytosed BODIPY-LDL. The lateral movement of the LDL between the PVs in living cells is observed following the sequence red, green, blue (5 s, $10 \mathrm{~s}, 15 \mathrm{~s})$ for merge 1; magenta, yellow, cyan (20 s, $25 \mathrm{~s}, 30 \mathrm{~s})$ for merge 2; and red and green ( $35 \mathrm{~s}$ and $40 \mathrm{~s}$ ) for merge 3 . In the insertions a, b and c, movement of BODIPY-LDL is observed in detail. PC: phase contrast. Bars, $10 \mu \mathrm{m}$. From Rivero \& Touz, unpublished. 
During evolution, eukaryotic cells differentiated, adapting to their increasingly complex environment by acquiring new abilities for nutrient uptake, internalization of growth factors, and entry of pathogens, among others. A complex array of endosomal compartments are preserved as modules that are able to perform specific functions in modern eukaryotic cells. In the case of less-evolved eukaryotes, the variety and simplicity of these systems is only starting to become apparent. Thus, Giardia possesses the endosomallysosomal system concentrated in the PVs, which may represent an ancient organellar system that later subdivided into compartments as early and late endosomes and lysosomes.

\section{Endosomal-lysosomal protein trafficking}

\subsection{Clathrin}

Clathrin-mediated endocytosis (CME) regulates many cell physiological processes, such as the internalization of growth factors and receptors, entry of pathogens, and synaptic transmission. Within the endocytic network, clathrin functions as a central organizing platform for coated pit assembly and dissociation via its terminal domain. As isolated from coated vesicles, clathrin is a trimer of 190-kDa heavy chains, each with an associated 25-kDa light chain forming a spiderlike molecule, the 'triskelion' (Kirchhausen, 2000). The unusual geometry of the triskelion allows it to assemble into regular polyhedral structures, the 'clathrin coats', which eventually give rise to clathrin-coated vesicles (CCVs). The dense protein coat of the CCV and its bristle-like morphology were first described by Roth \& Porter (1964), who noted the involvement of these vesicles in RME of yolk proteins in mosquito oocytes (Roth and Porter, 1964). The formation of CCVs occurs at the plasma membrane, trans-Golgi network and endosomes (Kirchhausen and Toyoda, 1993), and follows a sequence of coordinated steps, in which membrane invagination is coupled to growth of the clathrin lattice, leading to lattice closure and vesicle budding (Kirchhausen, 2000).

The Giardia genome encodes an ortholog of the clathrin heavy chain (CHC) (Morrison et al., 2007), and has a molecular weight of about $200 \mathrm{kDa}$, with three C-terminal clathrin repeats and one N-terminal propeller, according to a protein family database (Finn et al., 2008). Analysis of GlCHC expression showed that clathrin is expressed almost equally in both stages of the parasite and is located in close association with the PVs in trophozoites, and in the ESVs in immature cysts (Marti et al., 2003a; Marti et al., 2003b; Hehl and Marti, 2004; Gaechter et al., 2008). On the basis of these observations, it was suggested that recruitment of clathrin to late ESVs could serve to disperse large ESVs into smaller transport vesicles in response to the secretion signal (see below). The identification of a clathrin light chain (CL) ortholog in the GDB has so far been unsuccessful, probably because the sequences of CLs are not uniformly conserved among species. The differential expression of the CLs (e.g. one from yeast, two mammalian tissues with the presence of isoforms in mammalian neurons) might be associated with the high degree of specialization involved in clathrin vesicle trafficking. 
Several groups have presented evidence for a role of clathrin in endocytosis in Giardia (Touz et al., 2004; Hernandez et al., 2007; Gaechter et al., 2008; Rivero et al., 2010). However, neither typical membrane-associated clathrin lattices nor emerging clathrin-coated pits have been observed in this parasite. Instead, uncharacteristic coated pits were seen in close association with the PVs (Lanfredi-Rangel et al., 1998), suggesting that a distinct arrangement of clathrin might occur in this parasite. It is then possible that, as was observed in vitro (Zhang et al., 2007), clathrin may be organized in a hexagonal array, forming tubes instead of vesicles. Indeed, a different type of clathrin-coated transport carriers (TCs), consisting of larger tubular/vesicular structures having one or more clathrin-coated buds, have been identified (Polishchuk et al., 2006). These TCs travel long distances from the juxtanuclear area of the cell until they fuse with peripheral endosomes. The function of the TCs might be to mediate long-range distribution of mannose 6-phosphate receptors (MPR) and their cargo hydrolases to the peripheral cytoplasm (Puertollano et al., 2003). Similar to TCs in HeLa cells (Polishchuk et al., 2006), the tubules might not break down into CCVs en route to PVs in Giardia. In mammalian cells, it has been shown that TCs contain mannose-phosphate receptors, clathrin, Golgi-localizing Gamma-ear containing ARF-binding proteins (GGAs), and/or adaptor protein 1 (AP-1), and it was suggested that these might be uncoated during the TC-endosome fusion or could become integrated into the endosome membrane (Polishchuk et al., 2006). Possibly supporting this hypothesis, giardial clathrin and AP-1 were observed not only on ER-exist sites but also in PVs (Marti et al., 2003a; Marti et al., 2003b; Touz et al., 2004; Gaechter et al., 2008). As we said, the ER tubular-vesicular network apparently extends to and contacts the PVs in the periphery of the cell (Abodeely et al., 2009 b) but it was recently reported that no ER membranes invade the space occupied by PVs (Faso and Hehl, 2011). An explanation that reconciles these observations might be that at least some of the clathrin-dependent trafficking in Giardia involves tubular carriers that extend from the ER-exit sites to the peripheral cytoplasm until they meet with distally located PVs.

\subsection{Adaptor proteins}

The classic model for clathrin-dependent sorting comprises the participation of cargo receptors, adaptor heterotetramers and clathrin triskelia. Because clathrin has no affinity for biological membranes, its recruitment to membranes and capture of transmembrane cargo requires the action of clathrin-associated adaptor proteins (AP), which bind to clathrin through the amino-terminal domain of the CLH (Bonifacino and Traub, 2003). Among these adaptors are AP-1, AP-2 and AP-3, which comprise two large chains (one each of $\gamma / \alpha / \delta$ and $\beta 1-3$, respectively), one medium-sized chain ( $\mu 1-3)$, and one small chain $(\sigma 1-3)$ (Boehm and Bonifacino, 2002). These complexes are localized to different subcellular compartments, where they function in cargo selection (Boehm and Bonifacino, 2002). At least one of the large subunits in each AP complex $(\gamma / \alpha / \delta)$ mediates binding to the target membrane. The other large subunit, $\beta 1-3$, recruits clathrin through a 'clathrin-box' motif (Boehm and Bonifacino, 2001; Brodsky et al., 2001). The $\mu 1-3$ subunits are involved in the recognition of tyrosine-based, YXXØ signals (where $X$ represents any amino acid and $\varnothing$ indicates a residue 
with a bulky hydrophobic side chain), and combinations of $\alpha \sigma 2, \gamma \sigma 1$ and $\delta \sigma 3$ recognize dileucine-based, [DE]XXXI[LI] signals (Collins et al., 2002; Bonifacino and Traub, 2003). A fourth $\mathrm{AP}$ complex, $\mathrm{AP}-4$, is thought to be a component of a non-clathrin coat and to recognize a different type of signal (Burgos et al.). Besides the putative GlCHC, orthologs of two large, one medium, and one small subunit of each AP-1 and AP-2 are present in the Giardia genome. The colocalization of AP-1 with lysosomal proteins, its interaction with the $\mathrm{GlCHC}$, together with the observation that lysosomal protein trafficking is altered in $\mu 1$ depleted trophozoites, support the participation of this complex in the forward transport of proteins towards the PVs in Giardia (Touz et al., 2004). AP-1 also plays a central role during parasite differentiation, since $\mu 1$ depletion impairs encystation (Touz et al., 2004). On the other hand, AP-2 is localized to the PVs and plasma membrane in trophozoites and also neighboring the ESV in encysting cells (Rivero et al., 2010). AP-2 participates in RME and is crucial in the internalization of lipoproteins (Rivero et al., 2010). Although the $\beta 1-2$ and $\mu 2$ mRNA transcripts change little during the completion of the cell cycle (Marti et al., 2003b; Rivero et al., 2010), the role of the corresponding AP complexes appears essential for the adaptation of the parasite. AP-1 is not critical for Giardia trophozoite survival and multiplication, but it is necessary for cyst formation, acting indirectly in this process by transporting a transmembrane protein to the PVs (Touz et al., 2002b; Touz et al., 2003; Touz et al., 2004). In contrast, AP-2 is essential for Giardia growth and survival, being involved in the endocytosis of essential molecules (e.g., exogenous lipids) (Rivero et al., 2010) and in the fragmentation of ESVs into small transport vesicles containing cyst wall proteins during encystation (Rivero \& Touz, unpublished). The fast secretion and deposition of cyst wall material has been reported to involve clathrin- and dynamin-dependent breakup of ESVs into small vesicles targeted for the plasma membrane (Hehl and Marti, 2004; Gaechter et al., 2008). It is possible that this parasite requires the concerted action of clathrin and adaptors as well as accessory proteins at the time of cyst wall formation.

Taken together, these results support the hypothesis that Giardia possesses molecular mechanisms for lysosomal protein trafficking involving adaptor proteins similar to those of other eukaryotes. AP-1 and AP-2 appear to be the only two adaptors involved in lysosomal protein trafficking in Giardia, since there is no evidence of the participation of other adaptor proteins such as AP-3, AP-4, and monomeric adaptors (i.e., the GGAs). It has been suggested that the two prototypic Giardia AP complexes predict the point of separation of Giardia after the first coordinated round of gene duplications, resulting in an AP-3 and an AP-1/2/4 ancestor (Marti et al., 2003b). Phylogenetic reconstruction from comparative genomics has shown that all four $\mathrm{AP}$ complexes were present in the Last Common Eukaryotic Ancestor (LCEA), as was the F-COP subcomplex (Boehm and Bonifacino, 2001). However, the GGAs, which also exhibit homology to the ear region of the AP-1 $\gamma$ protein, are restricted to animal and fungal lineages (Field et al., 2007). Therefore, individuality of the species lineage and secondary loss are common characteristics in the evolutionary history of the adaptins. Secondary losses of adaptors can be observed in Drosophila melanogaster, Caeanorhabditis elegans, Saccharomyces cerevisiae and Schizosaccharomyces pombe, which lack the AP-4 complex (Field et al., 2007). In addition, comparative genomic and phylogenetic 
analyses of protozoan parasites have shown loss of the AP-3 complex in the species Theileria, Cryptosporidium parvum, and Babesia bovis, while Trypanosome brucei and Leishmania major lack AP-2 and AP-4, respectively (Nevin and Dacks, 2009). Thus, examination of the role of the reduced set of AP complexes in protozoa provides insight into the depths of our cellular history and highlights the importance of essential cell biology adaptations of the ancestral cellular organization.

\subsection{Lysosomal proteins}

Lysosomal integral membrane proteins (e.g., LAMP/LIMP family proteins) are transported to lysosomes by binding of their cytosolic motifs to AP complexes (Bonifacino and Lippincott-Schwartz, 2003). The carboxy-terminal $\beta$-sandwich domain of the $\mu$ subunits of AP-1, AP-2, and AP-3 binds directly to YXXØ-type sequences, while the $\gamma \sigma 1, \alpha \sigma 2$ and $\delta \sigma 3$ hemicomplexes bind to the [DE]XXXL[LI] sequences (Janvier et al., 2003; Doray et al., 2007). Although lysosomal integral membrane glycoproteins have not been identified in Giardia, it was reported that a cysteine protease termed ESCP (encystation-specific cysteine protease) is transported to the PVs through a tyrosine-based motif. This enzyme is homologous to cathepsin $C$ enzymes of higher eukaryotes and possesses a transmembrane domain and a YRPI motif within the cytoplasmic tail. ESCP localizes to the PVs in growing trophozoites and also to the plasma membrane in encysting cells (Touz et al., 2003). Deletion of the YRPI motif or suppression of $\mu 1$ mislocalizes this protein to the plasma membrane or to the ERexit sites, respectively (Touz et al., 2003; Touz et al., 2004).

Soluble acid hydrolases, on the other hand, are synthesized in the ER and transported to the Golgi complex, where their carbohydrate chains are modified by resident enzymes before delivery to lysosomes. In mammalian cells, the hydrolases are modified with mannose 6phosphate residues that function as recognition signals for MPRs in late Golgi compartments. In yeast, the vacuolar hydrolases lack mannose 6-phosphate, and the sorting receptor is the product of the VPS10 (Vacuolar Protein Sorting 10) gene. In both cases, however, sorting signals present in the cytosolic tails of the receptors interact with clathrin adaptors and direct packaging of the hydrolase-receptor complexes within CCVs or clathrincoated TCs (Ohno et al., 1995; Ohno et al., 1996). Recently, multi-ligand type-1 receptors Sortilin, SorCS1, SorCS2, SorCS3, and SorLA were discovered, containing an N-terminal Vps10p domain (Rezgaoui et al., 2001; Hermey, 2009). These are transmembrane proteins that convey Golgi-endosome transport and bind a number of unrelated ligands.

In Giardia, high hydrolase activity in the PVs has been implicated in protein degradation during growth (Lujan and Touz, 2003), encystation (Touz et al., 2002a; Touz et al., 2003) and excystation (Slavin et al., 2002). A family of three cysteine protease genes (CP1, CP2, and CP3) has been shown to encode members of the cathepsin B subgroup of the peptidase family C1 (Ward et al., 1997), and soluble CP2 has been found in PVs and ER of trophozoites (Ward et al., 1997; Abodeely et al., 2009a). Also, AcPh activity has been examined cytochemically, revealing communication of the PVs with the ER (Feely and Dyer, 1987; Lanfredi-Rangel et al., 1998; de Souza et al., 2004). Unlike AcPh in other eukaryotes, 
including protozoa, Giardia AcPh is a soluble protein that is transported from the ER-exit site to the PVs via AP-1 (Touz et al., 2004). It is thus possible that a specific receptor, possessing a function similar to the MPR or Vps10p, is involved in the trafficking of soluble hydrolases toward the PVs. Recent studies have identified a type-I membrane protein that interacts with AcPh and contains an YQII (YXXØ-type) motif in its cytosolic tail (Rivero \& Touz, unpublished). In silico analysis revealed that this protein (GDB: GL50803_28954) might be orthologous to the Vps10p receptor. Further biochemical studies on this putative receptor in both vegetative and encysting trophozoites and its participation in hydrolase delivery are necessary to elucidate the exact function of this protein.

Comparative analysis of lysosomal proteins present in Giardia and other cells reveals some intriguing differences. For instance, $\mathrm{AcPh}$ is soluble in Giardia but exists as a type-I membrane protein containing a YXXØ-type internalization sequence in cells as different as Leishmania and humans (Gottlieb and Dwyer, 1981; Waheed et al., 1988; Shakarian et al., 2002), with transport to the lysosome occurring through several cycles of plasma membrane internalization and recycling. In the lysosome of mammalian cells, the luminal domain of $\mathrm{AcPh}$ is processed and released in soluble form (Peters et al., 1990). Moreover, while the $\mathrm{AcPh}$ tail interacts with AP-2 in these cells, the lysosomal traffic of Giardia AcPh depends on AP-1 (Touz et al., 2004). Because much of the machinery involved in lysosomal trafficking is derived from a few protein families (where the various family members perform the same basic mechanistic function), the analysis of the similarities and differences between organisms might provide further insight into eukaryotic cell evolution.

As mentioned above, recent studies have shown that AP-2 participates in endocytosis of the Giardia Low-density lipoprotein Receptor-related Protein or LRP (Rivero et al., 2010). Giardia LRP is a type-I membrane protein, which shares the substrate-N-terminal binding domain and a FXNPXY-type endocytic motif with human LRP1. This receptor localizes predominantly to the ER but is also found in the PVs and plasma membrane in Giardia, and internalizes both low density lipoproteins (LDL) and chylomicrons as shown by in vitro studies. The FXNPXY motif of LRP was shown to bind directly to the $\mu 2$ subunit of AP-2, with this interaction being necessary for its proper localization, processing, and function.

One common characteristic of LDLR family members such as the LRPs is that they have at least one copy of the FXNPXY-like sequence in their cytosolic tail, which serves as the signal for endocytosis or as a binding element for adaptor proteins involved in signal transduction (Harris-White and Frautschy, 2005). In other eukaryotes, FXNPXY signals are recognized by the adaptor proteins Disabled homolog 2 (Dab2) and Autosomal Recessive Hypercholesterolemia (ARH), which contain a phosphotyrosine-binding (PTB) domain (Traub, 2009). However, no PTB-containing proteins such as Dab2 or ARH are encoded in the Giardia genome, supporting the idea that AP-2 might be the key endocytic adaptor in this parasite. Indeed, it has been shown by surface plasmon resonance and photoaffinity labeling that the FXNPXY-like motif binds to $\mu 2$ purified from bovine-brain-coated vesicles (Boll et al., 2002). Interestingly, in spite of the strong interaction found between ARH and 
LRP1 in an in vitro binding assay, the subcellular localization of LRP1 was not affected in the liver of ARH-deficient mice, whereas LDLR was found to be redistributed from intracellular localizations to the cell membrane (Jones et al., 2003). Thus, the importance of the availability of intracellular adaptor proteins might determine the specific cellular function of lipoprotein receptors. Since Giardia trophozoites do not have the capacity of de novo synthesis of cholesterol, its acquisition may depend on the internalization of chylomicrons from the host intestine by LRP. Moreover, because the trophozoites normally thrive in an environment where they never come in contact with LDL, it is possible that the binding of LDL to LRP represents a result of exaptation, thereby shifting the function of this protein allowing growth of parasites in culture medium.

\subsection{Accessory proteins}

\subsubsection{Dynamin}

Dynamins are large GTPases that belong to a protein superfamily, which are involved in many processes including clathrin-mediated endocytosis, clathrin-independent endocytosis, budding of transport vesicles, organelle division, cytokinesis and pathogen resistance (McNiven et al., 2000; Ochoa et al., 2000; Cao et al., 2003; Krueger et al., 2003). Substantial evidence indicates that dynamin oligomerization around the necks of endocytosing vesicles and subsequent dynamin-catalyzed GTP hydrolysis are responsible for membrane fission (Sweitzer and Hinshaw, 1998). Mammalian dynamins 1, 2 and 3 are the founder members of the dynamin family that, along with other large GTPases, possesses five identifiable domains: GTPase domain, middle domain, a lipid binding Pleckstrin-homology (PH) domain, GTPase Effector domain (GED) and C-terminal proline-arginine rich domain (PRD) (Hinshaw, 2000).

Recent studies indicate that Giardia possesses a single dynamin homolog (GIDRP, dynaminrelated protein), with the predicted protein containing the N-terminal GTPase domain (33219), the middle domain (230-523) and a C-terminal GED (628-719) (Marti et al., 2003b). The giardial dynamin (GIDRP, dynamin-related protein), has a PRD of 70 amino acids (538-608) (Gaechter et al., 2008). While the PRDs of dynamins are normally localized at the Cterminus, after the GED, in other eukaryotes, the PDR of the giardial dynamin is inserted between the middle domain and the GED. Interestingly, a typical $\mathrm{PH}$ domain that could mediate direct interaction with membrane lipids is missing.

GIDRP partially colocalizes with clathrin at the PVs and is necessary for endocytosis of plasma membrane proteins but not for fluid-phase endocytosis in Giardia trophozoites. Moreover, the expression of a mutant GIDRP, with reduced affinity for GTP and GDP, impaired endocytosis and resulted in enlarged PVs, indicative of blocked vesicular fission in these organelles. Also in these cells, GIDRP is detected at the ER, with only a minor proportion being present as a cytoplasmic pool. During encystations, however, both clathrin and GIDRP localize in part in the ESVs containing cyst wall material (Marti et al., 2003b; Hehl and Marti, 2004; Gaechter et al., 2008). Interestingly, matching the 
function observed in depleted- $\mu 2$ encysting trophozoites (Rivero et al., 2010), expression of a dominant-negative GIDRP affects the formation of small vesicles containing cyst wall proteins (CWPs) and blocks its exocytosis to form the cyst wall. Because close contact of the ESVs and PVs has been frequently reported (Marti et al., 2003b; Touz et al., 2003; Hehl and Marti, 2004; Gaechter et al., 2008), exchange of material between these two structures may occur, with the PVs finally acting as sorting organelles, probably by delivering the CWPs to the plasma membrane and/or returning other proteins to the ERexit site.

With the characterization of dynamic-like proteins from Homo sapiens, Drosophila melanogaster, Caenorhabditis elegans, yeast species, Arabidopsis thaliana, and Giardia, it may now be possible to reveal the many varied functions of the members of the dynamin superfamily in different organisms.

\subsubsection{Snares and rabs}

The basic steps underlying vesicle-mediated transport between the secretory and endocytic pathways are vesicle formation from a donor compartment, translocation of transport intermediates to a target organelle, tethering of transport intermediates with the target compartment, and, finally, the docking and fusion of vesicles with the membrane target. SNAREs (soluble N-ethylmaleimide-sensitive factor-attachment protein receptors) function in the final event of docking of vesicles with the target membrane, catalyzing the fusion of the opposing membranes. Compared to other organisms, Giardia has a relatively small number of SNARE proteins (Morrison et al., 2007; Elias et al., 2008). Seventeen putative SNAREs have been identified and partially characterized, with five representing QaSNAREs, five Qb-SNAREs, four Qc-SNAREs and three R-SNAREs. Although some of these SNAREs localize to the PV area, their function has not been investigated and has rather been inferred from the participation of their orthologs in other cells (Elias et al., 2008). For example, different gSNAREs are present in the PVs/plasma membrane area, Qa1, gQa3, gQa5, gQb2, gQb4, gQb5, and gR3, with the presence of three different gQa SNAREs suggested to be involved in distinct pathways such as exocytosis, endocytosis, and PV-PV fusion (Elias et al., 2008).

The Rab family GTPases regulates many steps of membrane traffic, including vesicle formation, vesicle movement along actin and tubulin networks, and membrane fusion. So far, over 70 members of the Rab family have been identified in mammalian cells, and each seems to have a characteristic intracellular localization and function. For instance, Rab5 plays roles in endocytosis, early endosome fusion, and caveolar vesicle targeting to early endosomes (Barbieri et al., 2000; Pelkmans et al., 2004), while Rab11 mediates slow endocytic recycling through recycling endosomes, and Rab4 mediates fast endocytic recycling directly from early endosomes (van der Sluijs et al., 1992; Ullrich et al., 1996). There is also a coordinated action of Rab5 and Rab7 in the sorting of cargo receptors by the retromer complex (Rojas et al., 2008). 
Similar to the SNAREs, there is a remarkable reduction in the members of this family, with only seven Rab proteins being present in the Giardia genome (Morrison et al., 2007). The giardial Rab11 has been localized to the PVs and cytoplasm but relocalizes to the ESVs during encystation (Morrison et al., 2007; Abodeely et al., 2009b). A Giardia Rab1 that localizes to ER-exit sites and PVs is also associated with the ESVs during encystation (Langford et al., 2002).

The question of whether Rabs and SNAREs participate in delivery of lysosomal proteins to the PVs remains unanswered and could be addressed in greater detail by functional analysis. Since each member of the Rab and SNARE family retains analogous biological functions in almost all the species analyzed, it will be interesting to determine whether, in Giardia, selective pressures might have been operating on distinguishing aspects of the lysosomal trafficking pathway, adapting the specificities of these proteins to accomplish their function.

\section{Conclusion}

Over the past few years, our understanding of the cell biological processes underlying the function of the PVs during Giardia growth and differentiation has advanced considerably. This is largely because of the complete sequencing and annotation of the Giardia genome, the development of transfection systems, highly sophisticated morphological analyses, and the identification of new parasite proteins that participate in endosomal/lysosomal trafficking. Endosomal/lysosomal trafficking pathways exhibit significant complexity and diversity in terms of morphology, function, and mechanisms among different organisms and cell types. As shown by several studies, part of the Giardia transport machinery is fairly well conserved. The existence in this organism of the GlCHC and dynamin, the presence of tetrameric adaptor proteins, and endosomal-lysosomal sorting motifs within cargo proteins, support an early acquisition of genes necessary for endosomal/lysosomal trafficking during eukaryotic evolution. Nevertheless, this parasite has experienced considerable diversification (Figure 5). The constraints of living under parasitic conditions have probably been the major driver for the reductive evolution of lysosome/endosome and Golgi compartments to maintain only those components that are essential for specific compartmentalization needs. For example, this parasite possesses only two of the four AP complexes, AP-1 and AP-2, which are involved in sorting signal recognition. No monomeric adaptor proteins have been identified so far. Also, reduced members of the Rab family are present and, although Rab11 has been associated with the PVs, further analysis will be necessary to assess Rab participation in membrane tethering and fusion to preserve the PVs identity. Similarly, investigation on the SNARE proteins closely associated with PVs will shed light on the mechanism of vesicle-vacuole fusion. Despite the progress in the field, it is clear that our molecular understanding of this complex situation remains far from complete. In particular, the current view that this parasite needs a reduced set of organelles and machinery to control nutrient uptake as well as 
degradation of intracellular proteins and endocytosed macromolecules is without precedent and raises several conceptual questions, some of which have been addressed in this chapter. In the last decade, PVs are emerging as sorting stations were molecules can be sorted and selected for plasma membrane or ER delivery. The future challenge will be to complete the pieces of this important puzzle to understand and unravel functions of the PVs and to throw light on the fundamental organizational principles of endosome/lysosome biogenesis in all eukaryotes.

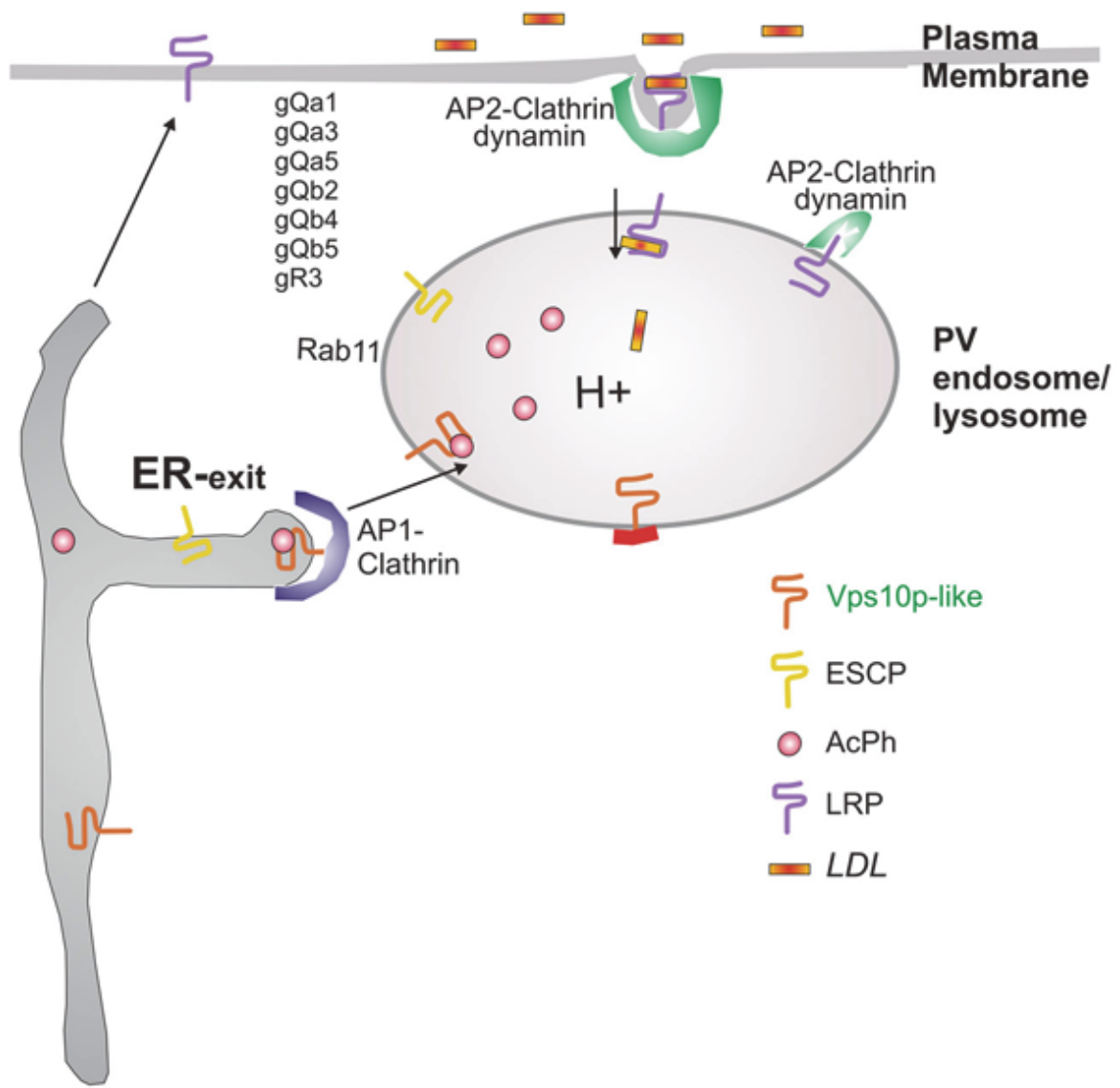

Figure 5. Schematic representation of lysosomal protein trafficking in growing Giardia trophozoites. From the ER-exit sorting site, the membrane protease ESCP is directed to the lysosome-like PVs in AP-1 and clathrin-coated vesicles. By the same pathway, the hydrolase AcPh is probably associated with the membrane receptor Vps10p and AP-1. AP-2 is involved in LDL/LRP endocytosis and PV delivery. The cytosolic proteins, clathrin and dynamin, are localized in the PVs. Rab11, and the SNAREs Qa1, gQa3, gQa5, gQb2, gQb4, gQb5, and gR3 may participate in vesicle trafficking to and/or from the PVs. H+ represents the acidic $\mathrm{pH}$ of the $\mathrm{PV}$ lumen. Unconfirmed protein participation is depicted in green. Modified from (Touz, 2011). 


\section{Author details}

Maria C. Touz

Instituto de Investigación Médica Mercedes y Martín Ferreyra, INIMEC - CONICET, National University of Cordoba, Córdoba, Argentina

\section{Acknowledgement}

I thank numerous colleagues for helpful suggestions. I am grateful to Nahuel Zamponi and Dr. Adriana Lanfredi-Rangel for the scanning microscopy images of Giardia trophozoite and cyst.

\section{References}

Abodeely, M., DuBois, K.N., Hehl, A., Stefanic, S., Sajid, M., DeSouza, W., Attias, M., Engel, J.C., Hsieh, I., Fetter, R.D., and McKerrow, J.H. (2009a). A contiguous compartment functions as endoplasmic reticulum and endosome/lysosome in Giardia lamblia. Eukaryot Cell 8, 1665-1676.

Abodeely, M., Dubois, K.N., Hehl, A., Stefanic, S., Sajid, M., Desouza, W., Attias, M., Engel, J.C., Hsieh, I., Fetter, R.D., and McKerrow, J.H. (2009b). A Contiguous Compartment Functions as Er and Endosome/Lysosome in Giardia Lamblia. Eukaryot Cell.

Adam, R.D. (2001). Biology of Giardia lamblia. Clin Microbiol Rev 14, 447-475.

Barbieri, M.A., Roberts, R.L., Gumusboga, A., Highfield, H., Alvarez-Dominguez, C., Wells, A., and Stahl, P.D. (2000). Epidermal growth factor and membrane trafficking. EGF receptor activation of endocytosis requires Rab5a. J Cell Biol 151, 539-550.

Barwick, R.S., Levy, D.A., Craun, G.F., Beach, M.J., and Calderon, R.L. (2000). Surveillance for waterborne-disease outbreaks--United States, 1997-1998. MMWR CDC Surveill Summ 49, 1-21.

Bockman, D.E., and Winborn, W.B. (1968). Electron microscopic localization of exogenous ferritin within vacuoles of Giardia muris. J Protozool 15, 26-30.

Boehm, M., and Bonifacino, J.S. (2001). Adaptins: the final recount. Mol Biol Cell 12, 29072920.

Boehm, M., and Bonifacino, J.S. (2002). Genetic analyses of adaptin function from yeast to mammals. Gene 286, 175-186.

Boll, W., Rapoport, I., Brunner, C., Modis, Y., Prehn, S., and Kirchhausen, T. (2002). The mu2 subunit of the clathrin adaptor AP-2 binds to FDNPVY and YppO sorting signals at distinct sites. Traffic 3, 590-600.

Bonifacino, J.S., and Lippincott-Schwartz, J. (2003). Coat proteins: shaping membrane transport. Nat Rev Mol Cell Biol 4, 409-414.

Bonifacino, J.S., and Traub, L.M. (2003). Signals for sorting of transmembrane proteins to endosomes and lysosomes. Annu Rev Biochem 72, 395-447. 
Brodsky, F.M., Chen, C.Y., Knuehl, C., Towler, M.C., and Wakeham, D.E. (2001). Biological basket weaving: formation and function of clathrin-coated vesicles. Annu Rev Cell Dev Biol 17, 517-568.

Burgos, P.V., Mardones, G.A., Rojas, A.L., daSilva, L.L., Prabhu, Y., Hurley, J.H., and Bonifacino, J.S. Sorting of the Alzheimer's disease amyloid precursor protein mediated by the AP-4 complex. Dev Cell 18, 425-436.

Cao, S., Yao, J., and Shah, V. (2003). The proline-rich domain of dynamin-2 is responsible for dynamin-dependent in vitro potentiation of endothelial nitric-oxide synthase activity via selective effects on reductase domain function. J Biol Chem 278, 5894-5901.

Collins, B.M., McCoy, A.J., Kent, H.M., Evans, P.R., and Owen, D.J. (2002). Molecular architecture and functional model of the endocytic AP2 complex. Cell 109, 523-535.

Dacks, J.B., and Doolittle, W.F. (2002). Novel syntaxin gene sequences from Giardia, Trypanosoma and algae: implications for the ancient evolution of the eukaryotic endomembrane system. J Cell Sci 115, 1635-1642.

de Souza, W., Lanfredi-Rangel, A., and Campanati, L. (2004). Contribution of microscopy to a better knowledge of the biology of Giardia lamblia. Microsc Microanal 10, 513-527.

Dobell, C. (1950). Samuel Hoole, translator of Leeuwenhoek's Select Works; with notes on that publication. Isis $41,171-180$.

Doray, B., Lee, I., Knisely, J., Bu, G., and Kornfeld, S. (2007). The gamma/sigma1 and alpha/sigma2 hemicomplexes of clathrin adaptors AP-1 and AP-2 harbor the dileucine recognition site. Mol Biol Cell 18, 1887-1896.

Elias, E.V., Quiroga, R., Gottig, N., Nakanishi, H., Nash, T.E., Neiman, A., and Lujan, H.D. (2008). Characterization of SNAREs determines the absence of a typical Golgi apparatus in the ancient eukaryote Giardia lamblia. J Biol Chem.

Elmendorf, H.G., Dawson, S.C., and McCaffery, J.M. (2003). The cytoskeleton of Giardia lamblia. Int J Parasitol 33, 3-28.

Faso, C., and Hehl, A.B. (2011). Membrane trafficking and organelle biogenesis in Giardia lamblia: Use it or lose it. Int J Parasitol.

Feely, D.E., and Dyer, J.K. (1987). Localization of acid phosphatase activity in Giardia lamblia and Giardia muris trophozoites. J Protozool 34, 80-83.

Field, M.C., Gabernet-Castello, C., and Dacks, J.B. (2007). Reconstructing the evolution of the endocytic system: insights from genomics and molecular cell biology. Adv Exp Med Biol 607, 84-96.

Finn, R.D., Tate, J., Mistry, J., Coggill, P.C., Sammut, S.J., Hotz, H.R., Ceric, G., Forslund, K., Eddy, S.R., Sonnhammer, E.L., and Bateman, A. (2008). The Pfam protein families database. Nucleic Acids Res 36, D281-288.

Fraser, D., Bilenko, N., Deckelbaum, R.J., Dagan, R., El-On, J., and Naggan, L. (2000). Giardia lamblia carriage in Israeli Bedouin infants: risk factors and consequences. Clin Infect Dis 30, 419-424.

Gaechter, V., Schraner, E., Wild, P., and Hehl, A.B. (2008). The single dynamin family protein in the primitive protozoan Giardia lamblia is essential for stage conversion and endocytic transport. Traffic 9, 57-71. 
Gerwig, G.J., van Kuik, J.A., Leeflang, B.R., Kamerling, J.P., Vliegenthart, J.F., Karr, C.D., and Jarroll, E.L. (2002). The Giardia intestinalis filamentous cyst wall contains a novel beta(1-3)-N-acetyl-D-galactosamine polymer: a structural and conformational study. Glycobiology 12, 499-505.

Gottlieb, M., and Dwyer, D.M. (1981). Leishmania donovani: surface membrane acid phosphatase activity of promastigotes. Exp Parasitol 52, 117-128.

Gu, F., Crump, C.M., and Thomas, G. (2001). Trans-Golgi network sorting. Cell Mol Life Sci $58,1067-1084$.

Harris-White, M.E., and Frautschy, S.A. (2005). Low density lipoprotein receptor-related proteins (LRPs), Alzheimer's and cognition. Curr Drug Targets CNS Neurol Disord 4, 469-480.

Hashimoto, T., Sanchez, L.B., Shirakura, T., Muller, M., and Hasegawa, M. (1998). Secondary absence of mitochondria in Giardia lamblia and Trichomonas vaginalis revealed by valyl-tRNA synthetase phylogeny. Proc Natl Acad Sci U S A 95, 6860-6865.

Hehl, A.B., and Marti, M. (2004). Secretory protein trafficking in Giardia intestinalis. Mol Microbiol 53, 19-28.

Hermey, G. (2009). The Vps10p-domain receptor family. Cell Mol Life Sci 66, 2677-2689.

Hernandez, Y., Castillo, C., Roychowdhury, S., Hehl, A., Aley, S.B., and Das, S. (2007). Clathrin-dependent pathways and the cytoskeleton network are involved in ceramide endocytosis by a parasitic protozoan, Giardia lamblia. Int J Parasitol 37, 21-32.

Hinshaw, J.E. (2000). Dynamin and its role in membrane fission. Annu Rev Cell Dev Biol 16, 483-519.

Huotari, J., and Helenius, A. (2011). Endosome maturation. EMBO J 30, 3481-3500.

Janvier, K., Kato, Y., Boehm, M., Rose, J.R., Martina, J.A., Kim, B.Y., Venkatesan, S., and Bonifacino, J.S. (2003). Recognition of dileucine-based sorting signals from HIV-1 Nef and LIMP-II by the AP-1 gamma-sigma1 and AP-3 delta-sigma3 hemicomplexes. J Cell Biol 163, 1281-1290.

Jarroll, E.L., Manning, P., Lindmark, D.G., Coggins, J.R., and Erlandsen, S.L. (1989). Giardia cyst wall-specific carbohydrate: evidence for the presence of galactosamine. Mol Biochem Parasitol 32, 121-131.

Jones, C., Hammer, R.E., Li, W.P., Cohen, J.C., Hobbs, H.H., and Herz, J. (2003). Normal sorting but defective endocytosis of the low density lipoprotein receptor in mice with autosomal recessive hypercholesterolemia. J Biol Chem 278, 29024-29030.

Kirchhausen, T. (2000). Clathrin. Annu Rev Biochem 69, 699-727.

Kirchhausen, T., and Toyoda, T. (1993). Immunoelectron microscopic evidence for the extended conformation of light chains in clathrin trimers. J Biol Chem 268, 10268-10273.

Krueger, E.W., Orth, J.D., Cao, H., and McNiven, M.A. (2003). A dynamin-cortactin-Arp2/3 complex mediates actin reorganization in growth factor-stimulated cells. Mol Biol Cell 14, 1085-1096.

Ladinsky, M.S., Wu, C.C., McIntosh, S., McIntosh, J.R., and Howell, K.E. (2002). Structure of the Golgi and distribution of reporter molecules at 20 degrees $C$ reveals the complexity of the exit compartments. Mol Biol Cell 13, 2810-2825. 
Lanfredi-Rangel, A., Attias, M., de Carvalho, T.M., Kattenbach, W.M., and De Souza, W. (1998). The peripheral vesicles of trophozoites of the primitive protozoan Giardia lamblia may correspond to early and late endosomes and to lysosomes. J Struct Biol 123, 225-235.

Langford, T.D., Silberman, J.D., Weiland, M.E., Svard, S.G., McCaffery, J.M., Sogin, M.L., and Gillin, F.D. (2002). Giardia lamblia: identification and characterization of Rab and GDI proteins in a genome survey of the ER to Golgi endomembrane system. Exp Parasitol 101, 13-24.

Lindmark, D.G. (1988). Giardia lamblia: localization of hydrolase activities in lysosome-like organelles of trophozoites. Exp Parasitol 65, 141-147.

Lujan, H.D., Mowatt, M.R., Conrad, J.T., Bowers, B., and Nash, T.E. (1995). Identification of a novel Giardia lamblia cyst wall protein with leucine-rich repeats. Implications for secretory granule formation and protein assembly into the cyst wall. J Biol Chem 270, 29307-29313.

Lujan, H.D., Mowatt, M.R., and Nash, T.E. (1997). Mechanisms of Giardia lamblia differentiation into cysts. Microbiol Mol Biol Rev 61, 294-304.

Lujan, H.D., and Touz, M.C. (2003). Protein trafficking in Giardia lamblia. Cell Microbiol 5, 427-434.

Manning, P., Erlandsen, S.L., and Jarroll, E.L. (1992). Carbohydrate and amino acid analyses of Giardia muris cysts. J Protozool 39, 290-296.

Marti, M., Li, Y., Schraner, E.M., Wild, P., Kohler, P., and Hehl, A.B. (2003a). The secretory apparatus of an ancient eukaryote: protein sorting to separate export pathways occurs before formation of transient Golgi-like compartments. Mol Biol Cell 14, 1433-1447.

Marti, M., Regos, A., Li, Y., Schraner, E.M., Wild, P., Muller, N., Knopf, L.G., and Hehl, A.B. (2003b). An ancestral secretory apparatus in the protozoan parasite Giardia intestinalis. J Biol Chem 278, 24837-24848.

McCaffery, J.M., and Gillin, F.D. (1994). Giardia lamblia: ultrastructural basis of protein transport during growth and encystation. Exp Parasitol 79, 220-235.

McNiven, M.A., Cao, H., Pitts, K.R., and Yoon, Y. (2000). The dynamin family of mechanoenzymes: pinching in new places. Trends Biochem Sci 25, 115-120.

Mellman, I., and Simons, K. (1992). The Golgi complex: in vitro veritas? Cell 68, 829-840.

Morrison, H.G., McArthur, A.G., Gillin, F.D., Aley, S.B., Adam, R.D., Olsen, G.J., Best, A.A., Cande, W.Z., Chen, F., Cipriano, M.J., Davids, B.J., Dawson, S.C., Elmendorf, H.G., Hehl, A.B., Holder, M.E., Huse, S.M., Kim, U.U., Lasek-Nesselquist, E., Manning, G., Nigam, A., Nixon, J.E., Palm, D., Passamaneck, N.E., Prabhu, A., Reich, C.I., Reiner, D.S., Samuelson, J., Svard, S.G., and Sogin, M.L. (2007). Genomic minimalism in the early diverging intestinal parasite Giardia lamblia. Science 317, 1921-1926.

Munro, S. (2004). Organelle identity and the organization of membrane traffic. Nat Cell Biol $6,469-472$.

Nash, T.E. (2002). Surface antigenic variation in Giardia lamblia. Mol Microbiol 45, 585-590.

Nevin, W.D., and Dacks, J.B. (2009). Repeated secondary loss of adaptin complex genes in the Apicomplexa. Parasitol Int 58, 86-94. 
Ochoa, G.C., Slepnev, V.I., Neff, L., Ringstad, N., Takei, K., Daniell, L., Kim, W., Cao, H., McNiven, M., Baron, R., and De Camilli, P. (2000). A functional link between dynamin and the actin cytoskeleton at podosomes. J Cell Biol 150, 377-389.

Ohno, H., Fournier, M.C., Poy, G., and Bonifacino, J.S. (1996). Structural determinants of interaction of tyrosine-based sorting signals with the adaptor medium chains. J Biol Chem 271, 29009-29015.

Ohno, H., Stewart, J., Fournier, M.C., Bosshart, H., Rhee, I., Miyatake, S., Saito, T., Gallusser, A., Kirchhausen, T., and Bonifacino, J.S. (1995). Interaction of tyrosine-based sorting signals with clathrin-associated proteins. Science 269, 1872-1875.

Pelkmans, L., Burli, T., Zerial, M., and Helenius, A. (2004). Caveolin-stabilized membrane domains as multifunctional transport and sorting devices in endocytic membrane traffic. Cell 118, 767-780.

Peters, C., Braun, M., Weber, B., Wendland, M., Schmidt, B., Pohlmann, R., Waheed, A., and von Figura, K. (1990). Targeting of a lysosomal membrane protein: a tyrosinecontaining endocytosis signal in the cytoplasmic tail of lysosomal acid phosphatase is necessary and sufficient for targeting to lysosomes. Embo J 9, 3497-3506.

Piva, B., and Benchimol, M. (2004). The median body of Giardia lamblia: an ultrastructural study. Biol Cell 96, 735-746.

Polishchuk, R.S., San Pietro, E., Di Pentima, A., Tete, S., and Bonifacino, J.S. (2006). Ultrastructure of long-range transport carriers moving from the trans Golgi network to peripheral endosomes. Traffic 7, 1092-1103.

Puertollano, R., van der Wel, N.N., Greene, L.E., Eisenberg, E., Peters, P.J., and Bonifacino, J.S. (2003). Morphology and dynamics of clathrin/GGA1-coated carriers budding from the trans-Golgi network. Mol Biol Cell 14, 1545-1557.

Reiner, D.S., Douglas, H., and Gillin, F.D. (1989). Identification and localization of cystspecific antigens of Giardia lamblia. Infect Immun 57, 963-968.

Rezgaoui, M., Hermey, G., Riedel, I.B., Hampe, W., Schaller, H.C., and Hermans-Borgmeyer, I. (2001). Identification of SorCS2, a novel member of the VPS10 domain containing receptor family, prominently expressed in the developing mouse brain. Mech Dev 100, 335-338.

Rivero, M.R., Miras, S.L., Quiroga, R., Ropolo, A.S., and Touz, M.C. (2011). Giardia lamblia low-density lipoprotein receptor-related protein is involved in selective lipoprotein endocytosis and parasite replication. Mol Microbiol 79, 1204-1219.

Rivero, M.R., Vranych, C.V., Bisbal, M., Maletto, B.A., Ropolo, A.S., and Touz, M.C. (2010). Adaptor Protein 2 Regulates Receptor-Mediated Endocytosis and Cyst Formation in Giardia lamblia. Biochem J.

Rojas, R., van Vlijmen, T., Mardones, G.A., Prabhu, Y., Rojas, A.L., Mohammed, S., Heck, A.J., Raposo, G., van der Sluijs, P., and Bonifacino, J.S. (2008). Regulation of retromer recruitment to endosomes by sequential action of Rab5 and Rab7. J Cell Biol 183, 513526.

Roth, T.F., and Porter, K.R. (1964). Yolk Protein Uptake in the Oocyte of the Mosquito Aedes Aegypti. L. J Cell Biol 20, 313-332. 
Shakarian, A.M., Joshi, M.B., Ghedin, E., and Dwyer, D.M. (2002). Molecular dissection of the functional domains of a unique, tartrate-resistant, surface membrane acid phosphatase in the primitive human pathogen Leishmania donovani. J Biol Chem 277, 17994-18001.

Slavin, I., Saura, A., Carranza, P.G., Touz, M.C., Nores, M.J., and Lujan, H.D. (2002). Dephosphorylation of cyst wall proteins by a secreted lysosomal acid phosphatase is essential for excystation of Giardia lamblia. Mol Biochem Parasitol 122, 95-98.

Sogin, M.L., Gunderson, J.H., Elwood, H.J., Alonso, R.A., and Peattie, D.A. (1989). Phylogenetic meaning of the kingdom concept: an unusual ribosomal RNA from Giardia lamblia. Science 243, 75-77.

Sun, C.H., McCaffery, J.M., Reiner, D.S., and Gillin, F.D. (2003). Mining the Giardia lamblia genome for new cyst wall proteins. J Biol Chem 278, 21701-21708.

Sweitzer, S.M., and Hinshaw, J.E. (1998). Dynamin undergoes a GTP-dependent conformational change causing vesiculation. Cell 93, 1021-1029.

Touz, M.C., Conrad, J.T., and Nash, T.E. (2005). A novel palmitoyl acyl transferase controls surface protein palmitoylation and cytotoxicity in Giardia lamblia. Mol Microbiol 58, 999-1011.

Touz, M.C., Gottig, N., Nash, T.E., and Lujan, H.D. (2002a). Identification and characterization of a novel secretory granule calcium-binding protein from the early branching eukaryote Giardia lamblia. J Biol Chem 277, 50557-50563.

Touz, M.C., Kulakova, L., and Nash, T.E. (2004). Adaptor protein complex 1 mediates the transport of lysosomal proteins from a Golgi-like organelle to peripheral vacuoles in the primitive eukaryote Giardia lamblia. Mol Biol Cell 15, 3053-3060.

Touz, M.C., Lujan, H.D., Hayes, S.F., and Nash, T.E. (2003). Sorting of encystation-specific cysteine protease to lysosome-like peripheral vacuoles in Giardia lamblia requires a conserved tyrosine-based motif. J Biol Chem 278, 6420-6426.

Touz, M.C., Nores, M.J., Slavin, I., Carmona, C., Conrad, J.T., Mowatt, M.R., Nash, T.E., Coronel, C.E., and Lujan, H.D. (2002b). The activity of a developmentally regulated cysteine proteinase is required for cyst wall formation in the primitive eukaryote Giardia lamblia. J Biol Chem 277, 8474-8481.

Touz, M.C., Rivero, M. R., Miras, S. L. and Bonifacino, J. S. (2011). Lysosomal Protein Trafficking in Giardia lamblia: Common and Distinct Features Front Biosci (Elite Ed). 4, 1898-1909.

Traub, L.M. (2009). Tickets to ride: selecting cargo for clathrin-regulated internalization. Nat Rev Mol Cell Biol 10, 583-596.

Ullrich, O., Reinsch, S., Urbe, S., Zerial, M., and Parton, R.G. (1996). Rab11 regulates recycling through the pericentriolar recycling endosome. J Cell Biol 135, 913-924.

van der Sluijs, P., Hull, M., Webster, P., Male, P., Goud, B., and Mellman, I. (1992). The small GTP-binding protein rab4 controls an early sorting event on the endocytic pathway. Cell 70, 729-740. 
Waheed, A., Gottschalk, S., Hille, A., Krentler, C., Pohlmann, R., Braulke, T., Hauser, H., Geuze, H., and von Figura, K. (1988). Human lysosomal acid phosphatase is transported as a transmembrane protein to lysosomes in transfected baby hamster kidney cells. Embo J 7, 2351-2358.

Ward, W., Alvarado, L., Rawlings, N.D., Engel, J.C., Franklin, C., and McKerrow, J.H. (1997). A primitive enzyme for a primitive cell: the protease required for excystation of Giardia. Cell 89, 437-444.

Zhang, F., Yim, Y.I., Scarselletta, S., Norton, M., Eisenberg, E., and Greene, L.E. (2007). Clathrin adaptor GGA1 polymerizes clathrin into tubules. J Biol Chem 282, 13282-13289. 